\title{
Radiated susceptibility analysis of single-wire transmission lines by means of Modified Stochastic Reduced-Order Modeling
}

\author{
Diego Bellan \\ Department of Electronics, Information and Bioengineering, Politecnico di Milano \\ Piazza Leonardo da Vinci 32, 20133 Milan \\ Italy
}

Received: July 14, 2020. Revised: August 12, 2020. Accepted: August 13, 2020. Published: August 14, 2020.

\begin{abstract}
This work investigates the statistical radiated susceptibility of an electrically-short transmission line (TL) consisting of a single wire over a ground plane. The angular parameters of the impinging plane wave and the height of the wire over the ground plane are modelled as random variables with Gaussian/Uniform distributions. The statistical properties of the current in the TL terminations (i.e., mean value, standard deviation, and cumulative distribution function) are derived through a properly defined numerical methodology consisting in a modified version of the conventional Stochastic Reduced-Order Model (SROM) approach. The proposed methodology consists in a straightforward approximation of the input continuous random variables with small-size discrete random variables. In contrast with conventional SROM, no numerical pre-processing is needed. The modified SROM proposed in this paper demonstrates high efficiency when compared with classical Monte Carlo approach. The proposed technique can be applied to the statistical analysis of much more complex systems whose input/output relationship requires a huge computational burden and for which the conventional Monte Carlo approach is not suitable.
\end{abstract}

Keywords-Radiated susceptibility, statistical analysis, stochastic modeling, transmission lines.

\section{INTRODUCTION}

$\mathrm{R}$ ADIATED susceptibility of transmission lines (TLs) is a canonical problem in electromagnetic compatibility (EMC). Usually the problem is tackled by assuming a plane wave impinging a TL with known geometrical/electrical parameters. A plane wave is characterized by four parameters, i.e., the amplitude and the three angles for polarization, elevation and azimuth. Under the assumption of deterministically known parameters, the analytical solution for currents/voltages induced in the TL terminations can be readily obtained for many TL configurations [1]. As far as practical applications are considered, however, a statistical approach to TL radiated susceptibility is of paramount importance since the parameters mentioned above are usually affected by uncertainty. Thus, a more appropriate modeling approach foresees a mathematical description of plane wave and TL parameters as random variables. As a consequence, currents/voltages in the TL terminations should be treated as random variables as well. In principle, since the analytical relationship between input parameters and termination currents/voltages is known, the statistical properties of the output variables can be analytically linked to the statistical properties of the input variables. However, the analytical form of the input/output relationship prevents the complete derivation of analytical results for the output statistics as functions of the input statistics. In the past literature such problem has been addressed and only partially solved in analytical forms for a simple canonical TL configuration consisting of an electrically-short single wire over a ground plane [2]. Even in this special case, the input/output relationship prevents the derivation of a complete statistical description in analytical and general terms. Thus, the problem must be tackled by resorting to numerical techniques. The most obvious approach is the so-called Monte Carlo approach, consisting in a huge number of repeated runs by assuming random behavior of the input parameters [3]. Such approach, however, is not optimal for the huge number of runs required. Moreover, in some cases a more complicated input/output relationship could result in heavy computational burden. Simpler and more effective numerical approaches, therefore, would be preferable and more suited to general applications. 
In recent years the Stochastic Reduced-Order Model (SROM) technique has been introduced to analyze the uncertainty propagation in stochastic systems [4]-[9]. Such approach is promising because if compared with other techniques (e.g., the stochastic collocation methods) is nonintrusive and does not require algebraic calculations. The main idea underlying SROM is the approximation in a statistical sense of the input (continuous) random variables by discrete random variables with a small number of possible values. Thus, SROM can be regarded as a smart and more efficient version of the Monte Carlo approach. SROM has been already successfully applied to another canonical EMC problem, i.e., crosstalk in three-conductor TLs with random electrical/geometrical parameters [10].

In this paper a modified version of SROM (i.e., MSROM) is introduced [11] and used to evaluate the mean value, the standard deviation, and the cumulative distribution function (CDF) of the current induced in the terminations of an electrically-short TL consisting in a single wire over a ground plane. The main difference between the conventional SROM and the proposed MSROM is in the procedure for the identification of the small number of values approximating the input random variables. In fact, while in the conventional SROM a numerical procedure is needed to extract such sample set and the related probabilities, in the MSROM proposed in this paper each random variable distribution is sampled analytically through the constraints provided by the statistical moments. Thus, no numerical pre-processing is needed. The methodology will be used for Gaussian and Uniform random variables, but it could be applied to any statistical distribution. Results obtained through MSROM are compared and validated against Monte Carlo results. The case study analyzed in this paper shows how the proposed methodology could be effectively used for the analysis of much more complicated input-output relationships. For example, the proposed methodology could be useful when the evaluation of the input/output relationship requires heavy and time-consuming numerical processing.

The paper is organized as follows. In Section II the problem of the radiated susceptibility of an electrically-short single wire over ground plane is described and the input/output relationship is formulated. In Section III the MSROM is described in detail and applied to the Gaussian and the Uniform distributions. In Section IV the proposed MSROM is numerically validated against the rough Monte Carlo approach. Moreover, interesting statistical properties of the TL radiated susceptibility are put into evidence and discussed. Conclusions and possible future research are discussed in Section V.

\section{PRoblem Statement}

Let us consider a two-conductor TL consisting in a horizontal wire with length $L$, radius $a$, and height $h$ over an ideal ground plane (see Fig. 1). Resistive loads $R_{L}$ and $R_{R}$ are considered at the left and right terminations, respectively. An impinging plane wave is considered, characterized by four parameters, i.e., the electric field amplitude $E_{0}$, the polarization angle $\eta$, and the direction of incidence identified by the angles $\theta$ and $\psi$. The

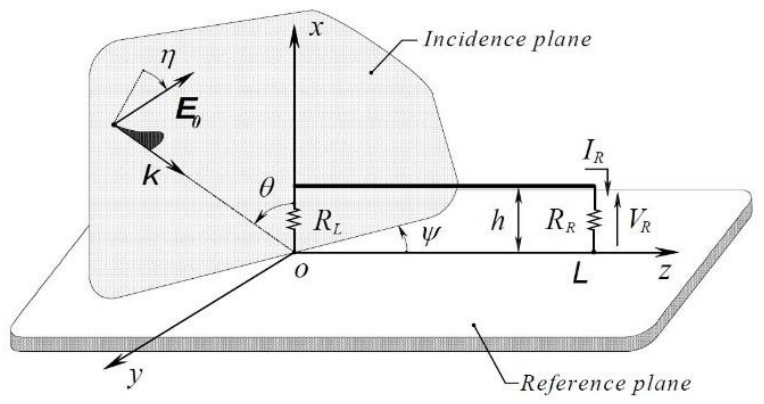

Fig. 1. Two-conductor TL consisting in one horizontal wire with length $L$ and height $h$ over a ground plane, excited by an impinging plane wave with electric field amplitude $E_{0}$, polarization angle $\eta$, and direction of incidence $\theta, \psi$. The current $I_{R}$ induced in the right load under the assumption of electrically short TL is given by (1).

induced currents in the left and right loads can be analytically evaluated as functions of the geometrical/electrical TL parameters and the four parameters of the impinging plane wave [1]. As far as an electrically short TL is considered, approximate and much simpler formulas can be derived for the load currents [2]. Indeed, under such assumption, it can be shown that the magnitude of the right-end load current can be written:

$$
\begin{aligned}
\left|I_{R}\right| & =\omega E_{0} \frac{2 h L}{c_{0}} \frac{R_{L}}{\left(R_{L}+R_{R}\right)} \mid \frac{1}{R_{L}}(\cos \vartheta \sin \psi \sin \eta+ \\
& +\cos \psi \cos \eta)-\frac{1}{z_{C}} \sin \vartheta \cos \eta \mid
\end{aligned}
$$

where $Z_{C}$ is the TL characteristic impedance

$$
Z_{C}=\frac{1}{2 \pi} \sqrt{\frac{\mu_{0}}{\varepsilon_{0}}} \log \frac{2 h}{a} .
$$

Notice that even if the simplified formula (1) is considered, the kind of relationship between the load current magnitude and the input parameters prevents a straightforward analytical derivation of the statistical properties of the load current when the input parameters are treated as random variables. In the next Section, a modified SROM approach is introduced such that the $\mathrm{CDF}$, the mean value, and the standard deviation of the load current magnitude (1) can be easily calculated by assuming a Gaussian or a Uniform distribution of a subset of input parameters (e.g., the three angular parameters of the impinging plane wave and the TL height).

\section{THE MODIFIED SROM}

SROM is a promising stochastic technique introduced quite recently in [4] and applied to EMC crosstalk analysis in [10]. Roughly speaking, the SROM approach consists in the approximation of the input continuous random variables with discrete random variables. Such discrete random variables are characterized by a small number of discrete values (i.e., the sample set), and by the probability assigned to each discrete value. The small size of the sample set results in reduced computational burden. Moreover, the probabilities assigned to 
the discrete values must be selected in order to approximate the statistics of the original random variables. Once the small-size sample set is selected, the input/output relationship is applied deterministically to the input sample set to obtain the related output values and the corresponding output probabilities. Thus, the SROM approach is nonintrusive and essentially consists in a smart version of the Monte Carlo approach since the input/output relationship is evaluated only for a small number of accurately selected input values.

The main drawback of the SROM approach outlined above is the numerical preliminary processing needed in order to accurately select the sample set of the input discrete random variables. In [4]-[10] such procedure is a completely numerical procedure oriented to select sample set and related probabilities such that the statistics of the input random variables are properly approximated. Moreover, in the general approach presented in [4]-[10] the input random variables can be statistically correlated.

In this paper, a simplified and modified SROM (MSROM) approach is introduced [11]. It consists in the discretization of the input random variables by selecting analytically (i.e., by avoiding the above mentioned numerical preliminary processing) a very small number of samples and the related probabilities such that the statistical moments of the original random variables are preserved. Moreover, in many cases of practical interests the input random variables can be considered as uncorrelated [12]-[14]. Therefore, once all the input random variables are discretized, the probability of each output event corresponding to specific values of the input random variables is given by the product of the probabilities of the involved samples of the input random variables. More specifically, by denoting with $n$ the number of input random variables, and with $k$ the number of discrete samples approximating each random variable, the number of possible combinations of input discrete values is given by the number of different permutations with repetitions $k^{n}$. The probability corresponding to each combination of discrete input values is given by the product of the probabilities of the involved input samples.

In this paper two random variable distributions are considered: the Gaussian random variable (already treated in [11]) and the Uniform random variable. The same approach, however, can be readily extended to arbitrary distributions.

\section{A. Gaussian distribution}

Let us consider a Gaussian random variable with mean value $\mu$ and variance $\sigma^{2}$. The minimum number of samples needed to provide a discretized form of such random variable is three. One sample located at $\mu$ with probability $p$, and two samples symmetrically positioned with respect to $\mu$ (i.e., located in $\mu \pm$ $\delta$ ) and with probability $\alpha p$, with $\alpha<1$ (Fig. 2a). Such sample distribution guarantees the mean value conservation. Further two constraints must be considered, i.e., the probability sum $p+\alpha p+\alpha p=1$ and the conservation of variance $\sigma^{2}$. The number of unknowns, however, is three, i.e., $\alpha, p$, and the location $\mu \pm \delta$ of the two symmetrical samples.

An arbitrary and reasonable choice is $\alpha=1 / 2$, such that $p+$ $\alpha p+\alpha p=1$ is fulfilled with $p=1 / 2$. The variance of such

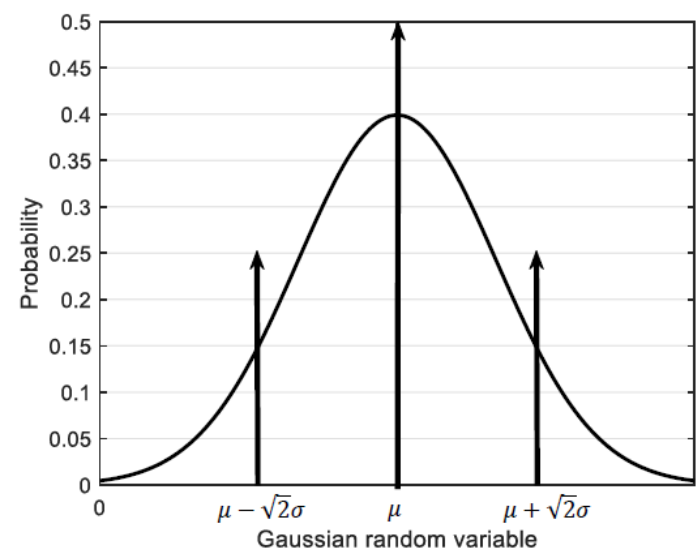

(a)

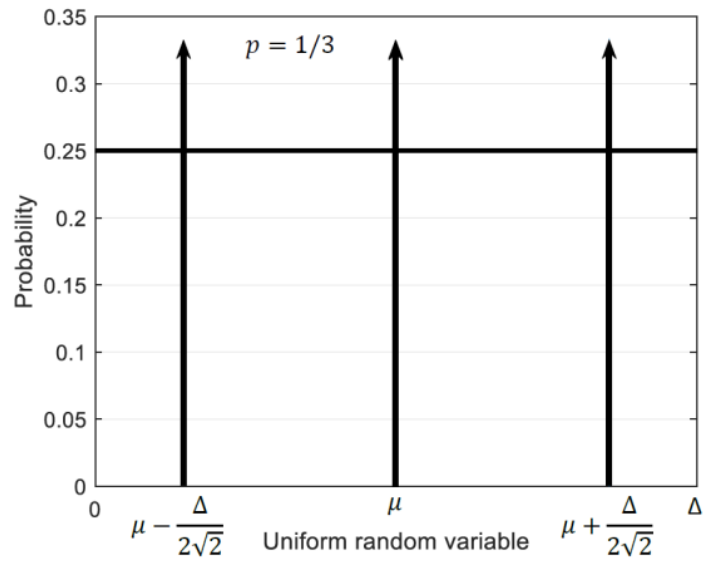

(b)

Fig. 2. Discrete 3-sample approximation of (a) Gaussian and (b) Uniform random variables with conservation of mean value and variance.

discrete variable is set equal to $\sigma^{2}[15]$ :

$$
(-\delta)^{2} \alpha p+\delta^{2} \alpha p=\sigma^{2}
$$

Therefore, from (3) the location of the two symmetrical samples is given by

$$
\delta=\sqrt{2} \sigma
$$

Thus, as outlined above, each Gaussian random variable is approximate by a discrete random variable with only three samples (i.e., $k=3$ ). One sample with probability $p=1 / 2$ located in $\mu$, and two samples with probabilities $\alpha p=1 / 4$ located in $\mu \pm \sqrt{2} \sigma$. The same discretization is performed for all the $n_{G}$ input random variables with Gaussian distribution.

\section{B. Uniform distribution}

Let us consider a Uniform random variable with mean value $\mu$ and variance $\sigma^{2}=\Delta^{2} / 12$, where $\Delta$ is the distribution range (Fig. 2b). The corresponding discrete random variable with minimum sample size consists of three samples with equal probability $p=1 / 3$, such that the probability sum is equal to one. The central sample is located in $\mu$, whereas the two symmetrical samples are located in $\mu \pm \delta$. The variance 
constraint provides [15]:

$$
(-\delta)^{2} p+\delta^{2} p=\sigma^{2}
$$

By taking into account $p=1 / 3$ and $\sigma^{2}=\Delta^{2} / 12$ from (5) we obtain:

$$
\delta=\sqrt{\frac{3}{2}} \sigma=\frac{\Delta}{2 \sqrt{2}}
$$

Thus, each Uniform random variable is approximated by a discrete random variable with only three samples (i.e., $k=3$ ), with equal probability $p=1 / 3$. One sample is located in $\mu$, and two samples are located in $\mu \pm \frac{\Delta}{2 \sqrt{2}}$. The same discretization is performed for all the $n_{U}$ input random variables with Uniform distribution.

By denoting as $n=n_{G}+n_{U}$ the total number of input random variables, the number of possible combinations of discrete input values is $3^{n}$, and the related probability is the product of the probabilities of each involved sample. For example, if $n=2$ the number of possible combinations of input values is $3^{2}=9$. The probability of each combination is given by $p_{1} p_{2}$, where $p_{1}$ and $p_{2}$ can take the values $1 / 2$ and $1 / 4$ in case of Gaussian random variable, or $1 / 3$ in case of Uniform random variable. Each probability provided by the product $p_{1} p_{2}$ is assigned to the output variable which is deterministically evaluated at the corresponding input samples.

\section{NUMERICAL VALIDATION}

The modified SROM approach outlined in Section III was validated for the radiated susceptibility problem described in Section II. The input/output relationship is given by the load current magnitude (1). The deterministic parameters were selected as $L=1 \mathrm{~m}, a=0.5 \mathrm{~mm}, R_{L}=R_{R}=50 \Omega, f=$ $1 \mathrm{MHz}, E_{0}=1 \mathrm{~V} / \mathrm{m}$, whereas the input parameters selected as random variables are the three angles $\eta, \theta$, and $\psi$ of the impinging plane wave, and the height $h$ of the wire.

A first set of simulations was performed by assuming the four random variables as Gaussian, and by approximating each continuous distribution as a discrete three-sample random variable as outlined in Subsection IIIA. The number of combinations of input values is $3^{4}=81$. The probability related to each of the 81 combinations is evaluated as the product $p_{\eta} p_{\theta} p_{\psi} p_{h}$, where each elemental probability can take the value $1 / 2$ or $1 / 4$ according to the involved sample (central or side sample of the discrete distribution). The product probability is assigned to the value of the load current evaluated through (1) for the corresponding values of the Gaussian samples. The numerical analysis is oriented to study the behavior of the TL radiated susceptibility as a function of the impinging direction of the plane wave. To this aim, the mean value $\mu_{\psi}$ of the incident angle $\psi$ is treated as a parameter ranging from 0 to $180^{\circ}$ (i.e., the independent variable in Figs. $3)$. For each value of $\mu_{\psi}$ the statistical analysis outlined above was performed by assuming mean values $\mu_{\eta}=0, \mu_{\vartheta}=45^{\circ}$,

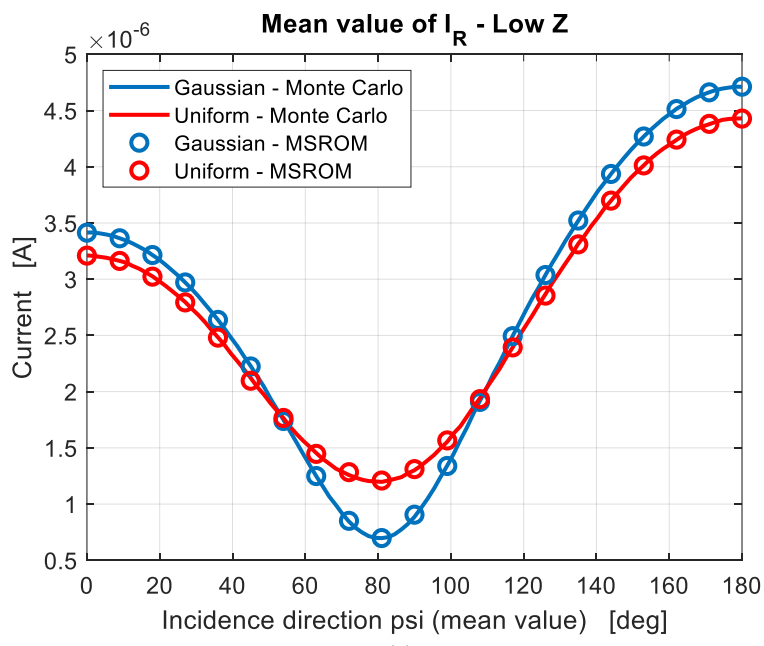

(a)

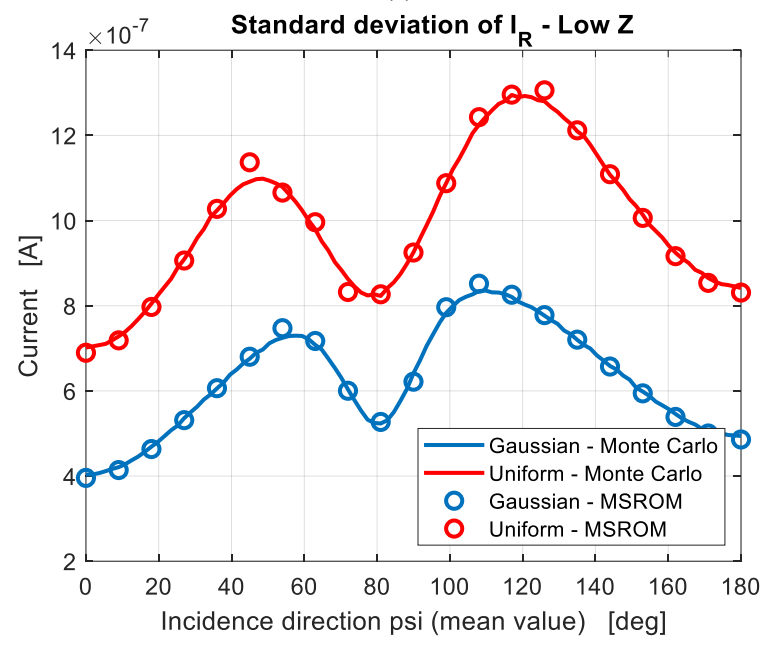

(b)

Fig. 3. Mean value (a) and standard deviation (b) of the load current magnitude as functions of the mean value of the incidence angle $\psi$, by assuming the three plane-wave angles and the wire height $h$ as random variables with Gaussian (blue) and Uniform (red) distributions. Comparison between Monte Carlo (solid lines) and the proposed modified SROM (MSROM (markers). Low-impedance loads are considered, i.e., $R_{L}=R_{R}=50 \Omega$.

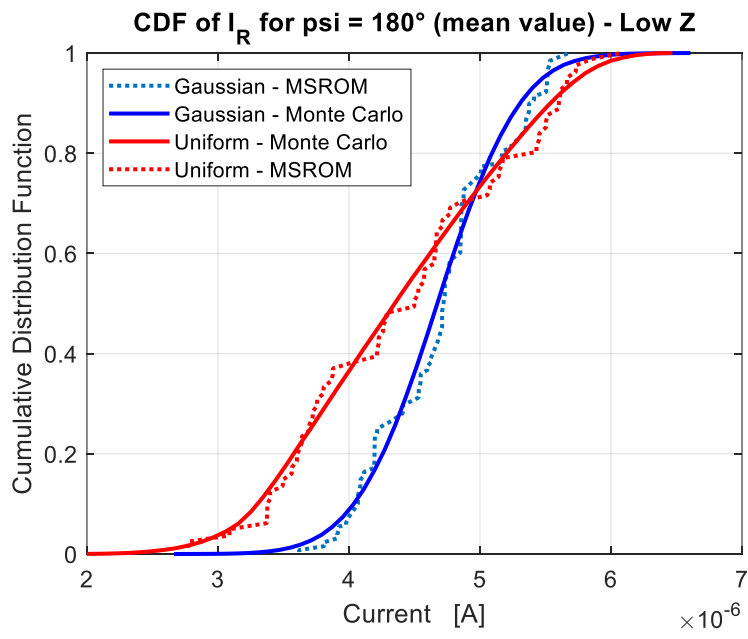

Fig. 4. CDF of the load current with Gaussian (blue) and Uniform (red) distributions for the input random variables. Monte Carlo (solid lines) is compared with the MSROM method (markers) in case of lowimpedance loads. 
$\mu_{h}=10 \mathrm{~mm}$, and standard deviations $\sigma_{\psi}=\sigma_{\eta}=\sigma_{\vartheta}=10^{\circ}$, $\sigma_{h}=1 \mathrm{~mm}$. Notice that from (2) (where $\mu_{h}$ is considered for h) we obtain $Z_{C}=221 \Omega$. Thus, the selected loading condition $R_{L}=R_{R}=50 \Omega$ can be considered as a low-impedance load. By taking into account also the mean values of the angular variables, the dominant contribution in (1) is provided by the function $\cos \psi$. This explains the behavior of the load current mean value represented in Fig. 3a where the solid blue curve represents the Monte Carlo results with $10^{5}$ repeated runs for each value of $\mu_{\psi}$, whereas the blue markers show the results of the proposed modified SROM (MSROM). In Fig. $3 \mathrm{~b}$ the comparison for the standard deviation of the load current is reported (blue curves). In Fig. 4 the CDF of the load current is reported for the case $\mu_{\psi}=180^{\circ}$ (blue curves). This corresponds to the maximum value reached in Fig. 3a, where the additive terms in (1) provide contributions with the same sign.

A second set of simulations was performed by assuming Uniform distribution for the four random variables, and introducing the corresponding discrete approximation according to Subsection IIIB. The mean values of the random variables were unchanged, whereas the range $\Delta$ of each random variable was assumed six times the standard deviation of the corresponding Gaussian distribution in the previous case. This choice allows the comparison between Uniform and Gaussian distributions in case of random variables with similar range. Since the Uniform distribution is spread on the whole range, we expect lower peaks and variability for the load current. This is confirmed by the red curves in Fig. 3a. On the contrary, for the same reason we expect higher standard deviation of the load current, and this is confirmed by the red curves in Fig. 3b. A higher spread in the Uniform case is also confirmed by the CDF (red curves) in Fig. 4.

A third set of simulations was performed by assuming $R_{L}=$ $1 k \Omega$ instead of $R_{L}=50 \Omega$. This case can be seen as a highimpedance load case when compared with $Z_{C}$. This choice makes the impact of the function $\cos \psi$ in (1) much lower, and therefore a more regular behavior is obtained in Fig. 5a for the mean value of the load current in the Gaussian and Uniform cases. In Fig. 5b a higher standard deviation due to the spread of the Uniform distribution is confirmed. A similar consideration holds for the CDFs of the load current in the case $\mu_{\psi}=180^{\circ}$ represented in Fig. 6 .

A further set of simulations was performed by assuming horizontal polarization instead of vertical polarization of the impinging waves. In fact, in the above simulations, the mean value of the polarization angle was $\mu_{\eta}=0$. On the contrary, in this final set of simulations the mean value of the polarization angle was $\mu_{\eta}=90^{\circ}$. The behavior of the mean value and the standard deviation of the induced current are represented in Figs. 7a-7b (low-impedance case) and Figs. 8a-8b (highimpedance case). Horizontal polarization entails the term $\sin (\eta)$ is dominating over the term $\cos (\eta)$ in (1). This explains the $\sin (\psi)$ behavior in Fig. 7a. Such behavior is less apparent in Fig. 8a since the high $R_{L}$ value attenuates the $\sin (\psi)$ term in (1). Finally, it is worth noticing that the behavior of the standard

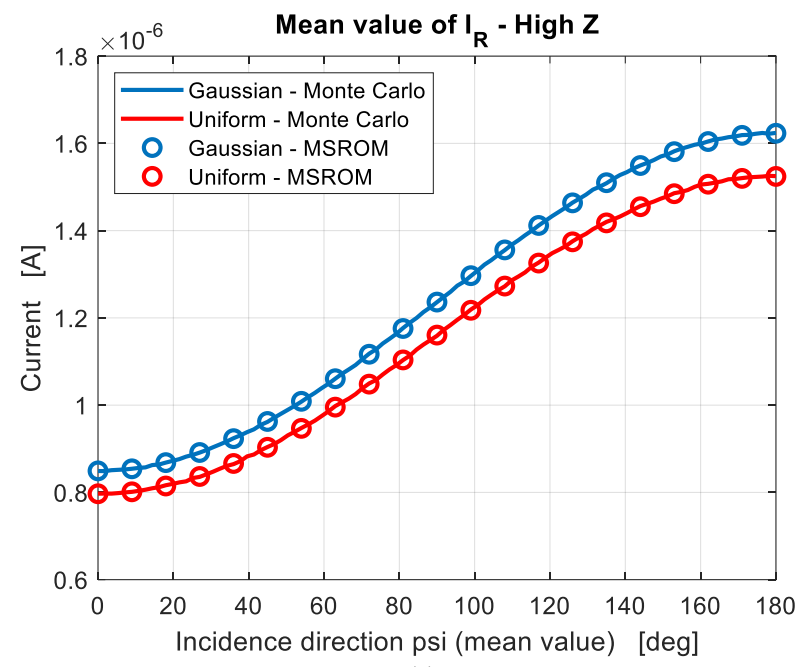

(a)

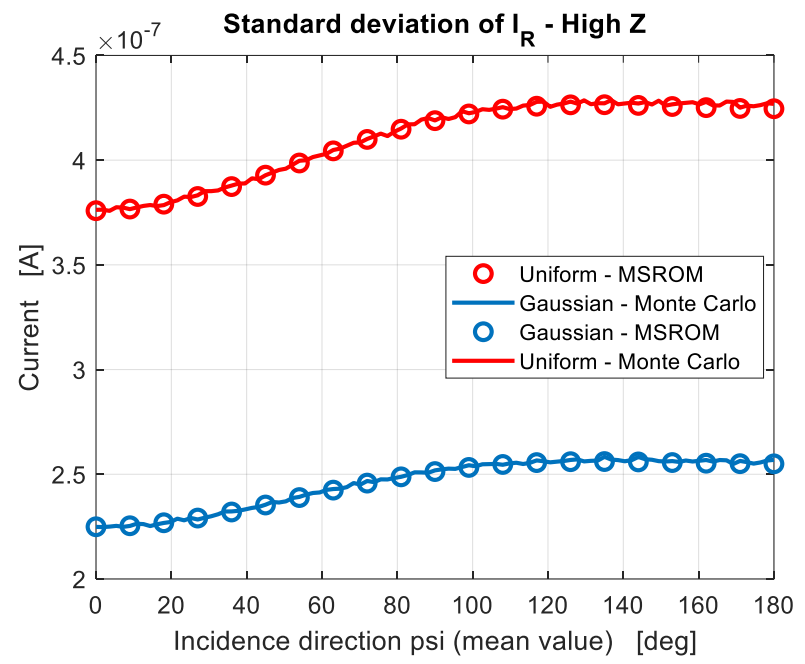

(b)

Fig. 5. Same as Fig. 3, with high-impedance load, i.e., $R_{L}=1 k \Omega$.

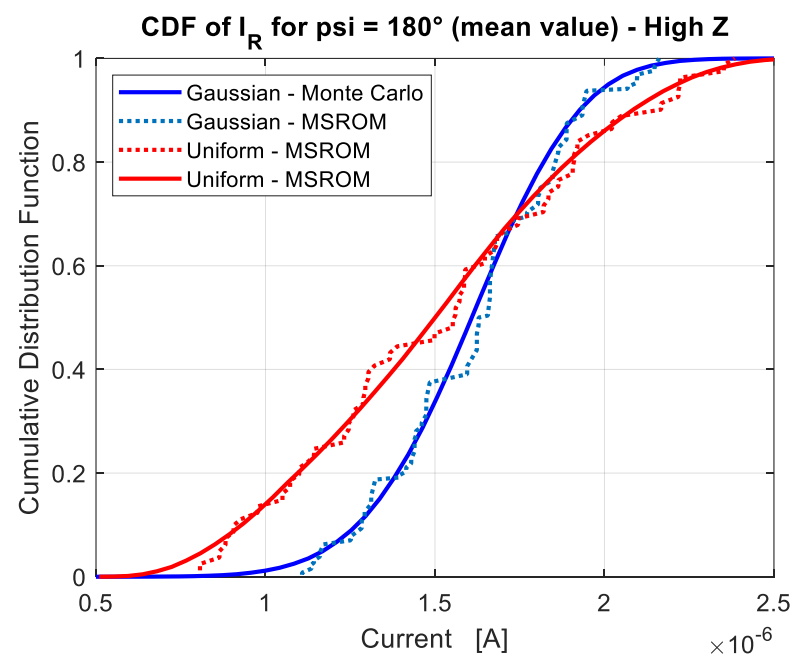

Fig. 6. CDF of the load current with Gaussian (blue) and Uniform (red) distributions for the input random variables. Monte Carlo (solid lines) is compared with the MSROM method (markers) in case of highimpedance load. 


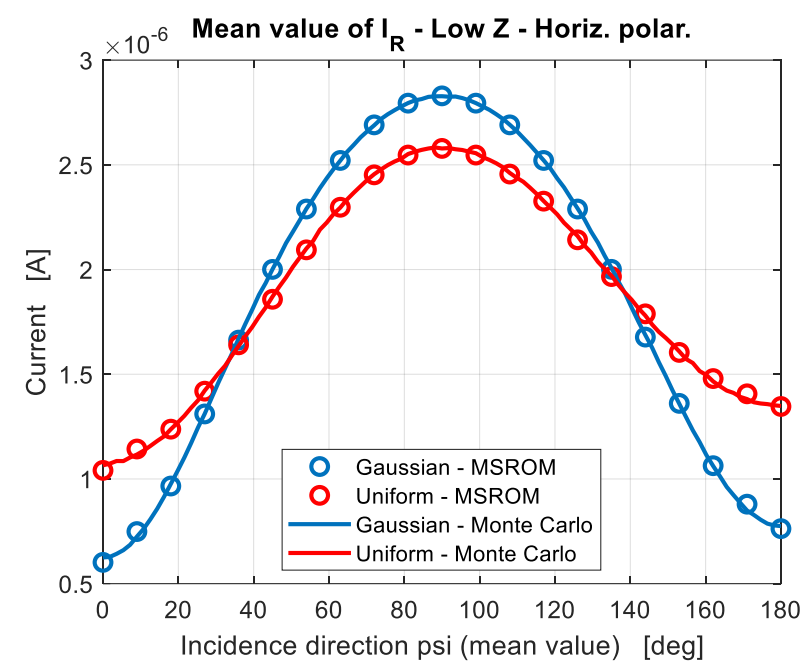

(a)

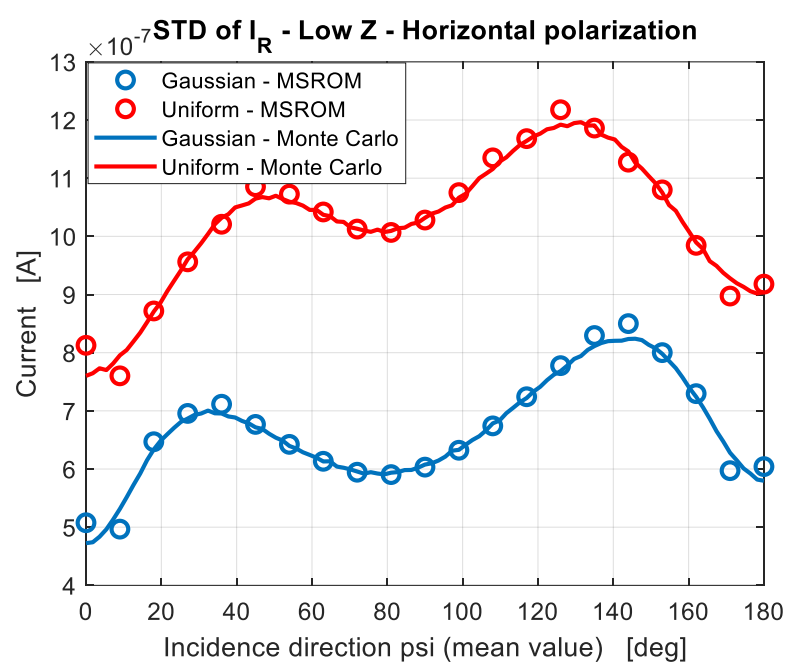

(b)

Fig. 7. Same as Fig. 3 but assuming horizontally polarized (i.e., $\mu_{\eta}=$ $90^{\circ}$ ) instead of vertically polarized impinging plane waves.

deviations in Figs. $7 \mathrm{~b}$ and $8 \mathrm{~b}$ show worse agreement between MSROM and Monte Carlo with respect to Figs. 3b and 5b. This is related to the specific condition of a horizontally polarized wave impinging on a perfectly conducting ground plane. As a whole, however, all the above simulations provide a satisfactory validation of the proposed MSROM technique.

\section{DISCUSSION AND CONCLUSION}

Approximating continuous random variables with small-size discrete random variables has proven to be a very powerful technique to obtain the statistical properties of an output variable when the relationship with input variables prevents analytical derivations. Gaussian and Uniform distributions were approximated by means of only three samples, selected by preserving the mean value and the variance of the underlying distributions. The proposed MSROM, in contrast with the conventional SROM, allows a simple and straightforward identification of the values of the discrete random variables and the related probabilities. No preliminary numerical processing

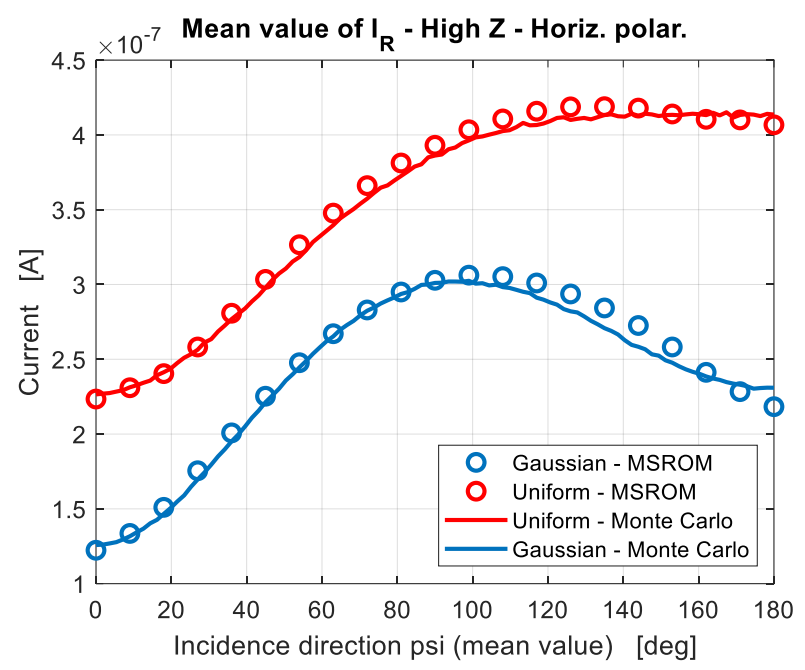

(a)

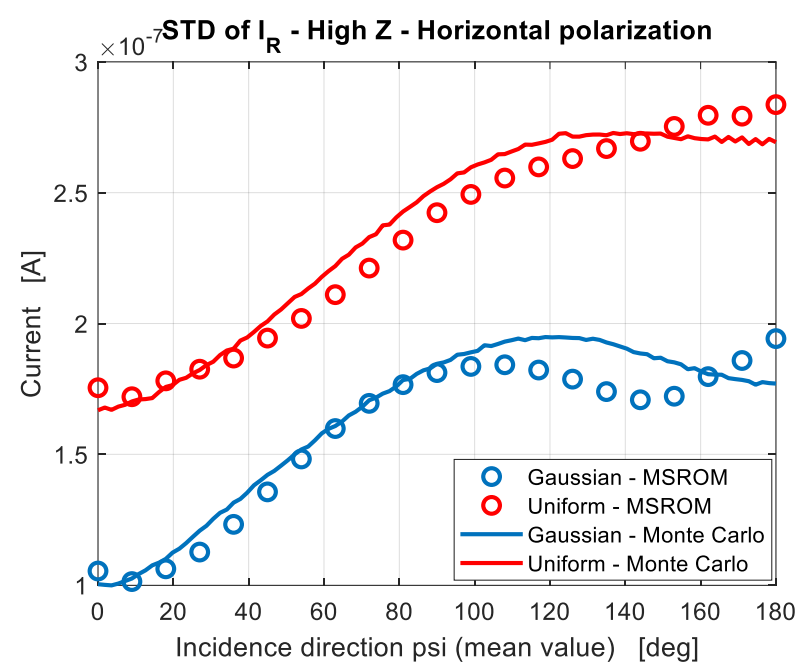

(b)

Fig. 8. Same as Fig. 5 but assuming horizontally polarized (i.e., $\mu_{\eta}=$ $90^{\circ}$ ) instead of vertically polarized impinging plane waves.

of data is needed. It is worth noticing that the proposed approach, in the present form, cannot handle the case of correlated input variables. In fact, the probability of each output value was evaluated by a simple multiplication of the corresponding input probabilities. This point will be investigated in future research.

The obtained results in terms of mean value, standard deviation, and CDF have shown astonishing agreement with the rough Monte Carlo approach. The advantage of the proposed approach, however, would be better appreciated in case of complex input-output relationships requiring heavy computational burden. This would be the case of the numerical simulation of a complex system where the parameters are affected by uncertainty. In case each simulation run requires long processing time the Monte Carlo method is not feasible. In such case the proposed MSROM would be a useful alternative since a small number of runs would provide a satisfactory statistical description of the output variables. 


\section{References}

[1] C. R. Paul, Analysis of Multiconductor Transmission Lines, $2^{\text {nd }}$ Edition, Wiley, 2007.

[2] D. Bellan and S. A. Pignari, "A probabilistic model for the response of an electrically short two-conductor transmission line driven by a random plane wave field," IEEE Trans. Electromagn. Compat., vol. 43, pp. 130-139, 2001.

[3] C. P. Robert and G. Casella, Introducing Monte Carlo methods wit R, Springer, 2009.

[4] M. Grigoriu, "Reduced order models for random functions. Application to stochastic problems," Appl. Math. Model., vol. 33, no. 1, pp. 161-175, 2009.

[5] J. E. Warner, W. Aquino and M. Grigoriu, "Stochastic reduced order models for inverse problems under uncertainty", Comput. Methods Appl. Mech. Eng., vol. 285, pp. 488-514, Mar. 2015.

[6] R. V. Field, M. Grigoriu and J. M. Emery, "On the efficacy of stochastic collocation stochastic Galerkin and stochastic reduced order models for solving stochastic problems", Probab. Eng. Mech., vol. 41, pp. 60-72, Jul. 2015.

[7] J. E. Warner, M. Grigoriu and W. Aquino, "Stochastic reduced order models for random vectors: application to random eigenvalue problems", Probab. Eng. Mech., vol. 31, pp. 1-11, Jan. 2013.

[8] M. Grigoriu, "Solution of linear dynamic systems with uncertain properties by stochastic reduced order models", Probab. Eng. Mech., vol. 34, pp. 168-176, Oct. 2013.
[9] S. Sarkar, J. E. Warner, W. Aquino and M. Grigoriu, "Stochastic reduced order models for uncertainty quantification of intergranular corrosion rates", Corros. Sci., vol. 80, pp. 257-268, Mar. 2014.

[10]Z. Fei, Y. Huang, J. Zhou, and Q. Xu, "Uncertainty quantification of crosstalk using stochastic reduced order models," IEEE Trans. Electromag. Compat., vol. 59, no. 1, pp. 228-239, 2017.

[11]D. Bellan and S. A. Pignari, "Investigation of Transmission Line Response to Random Plane Waves Through Stochastic Reduced Order Modeling," in Proc. of Progress In Electromagnetics Research Symposium (PIERS 2017), St. Petersburg, Russia, Nov. 22-25, 2017.

[12]D. Bellan and S. A. Pignari, "Efficient estimation of crosstalk statistics in random wire bundles with lacing cords," IEEE Trans. on Electromagn. Compat., vol. 53, no. 1, pp. 209-218, 2011.

[13] D. Bellan and S. A. Pignari, "Statistical superposition of crosstalk effects in cable bundles," China Communications, vol. 10, no. 11, pp. 119-128, 2013.

[14]D. Bellan, "Characteristic Function of Fundamental and Harmonic Active Power in Digital Measurements Under Nonsinusoidal Conditions," International Review of Electrical Engineering, vol. 10, no. 4, pp. 520-527, 2015.

[15] A. Papoulis, Probability Random Variables and Stochastic Processes, New York, NY, USA, McGraw-Hill, 1991.

\section{Creative Commons Attribution License 4.0 (Attribution 4.0 International, CC BY 4.0)}

This article is published under the terms of the Creative Commons Attribution License 4.0

https://creativecommons.org/licenses/by/4.0/deed.en_US 